\title{
DESENVOLVIMENTO DE SISTEMÁTICA DE DOAÇÃO DE CÓRNEAS EM INSTITUIÇÃO HOSPITALAR DE CARDIOLOGIA DE SÃO PAULO
}

\author{
Development of a cornea donation systematic in a São Paulo Cardiology Institution
}

\author{
Christiane Pereira Martins Casteli, Andréa Cotait Ayoub, César Augusto Guimarães Marcelino, Carine Cristiane Fusco, Mara \\ Nogueira Araújo.
}

\begin{abstract}
RESUMO
Objetivo: O estudo objetivou desenvolver uma sistemática para a doação intra-hospitalar de córneas visando favorecer o aumento de notificações de potenciais doadores em benefício da sociedade. Método: Trata-se de um relato de experiência cuja metodologia foi realizar o diagnóstico situacional referente ao número de óbitos, notificações e doadores efetivos de córneas, capacitar os colaboradores das áreas críticas para atuarem no processo de doação de córneas, estabelecer fluxograma para notificação e criar indicadores para levantamento, mensuração e análise dos resultados. Resultado: No diagnóstico situacional foi apurada baixa efetivação de notificação e doação de córneas: dos 438 (100\%) óbitos, 87 (20\%) foram notificados à OPO, efetivando-se a doação em apenas 14 (16\%). Adotou-se como estratégia a implementação de um fluxograma para notificação de potenciais doadores de córneas e capacitação de 76 (39,58 \%) profissionais das áreas críticas, com margem de erro após a aula de 3,9\% (30), demonstrando que as dúvidas foram minimizadas por meio do conteúdo exposto. Para mensurar mensalmente o número de óbitos, notificações e doações de córneas foi elaborado um instrumento de Busca Ativa compartilhado com as chefias médicas e de enfermagem envolvidas nesse processo. Conclusão: O estabelecimento do fluxograma interno, o envolvimento e a contribuição efetiva da equipe de enfermagem a partir da capacitação em serviço refletiram-se no aumento do número de notificações, alcançando 275 (86,2\%), na queda do número de não-notificações em 49,4\% (44) e no aumento da doação de córneas em $50 \%$, disponibilizando-se à sociedade 21 córneas.
\end{abstract}

Descritores: Transplante, Capacitação em serviço, Enfermagem.

\section{Instituição:}

Organização de Procura de Órgãos, Instituição Dante Pazzanese de Cardiologia, São Paulo, SP

\section{Correspondência:}

Christiane Pereira Martins Casteli

Av. Dr. Dante Pazzanese, 500. Pq. Ibirapuera. São Paulo-São Paulo. CEP: 04012-909. Diretoria de Enfermagem $-7^{\circ}$ andar. Prédio Administrativo (Torre).

Tel: (11) 50856000 (ramal: 6020/6076)

E-mail: chrispereiramartins@gmail.com

\section{INTRODUÇÃO}

A Portaria No 1262, de 16 de junho de 2006,1 determina como uma das atribuições a ser desenvolvida pela Organização de Procura de Órgãos (OPO) nos hospitais públicos, privados e filantrópicos com mais de 80 leitos a promoção de um programa de educação continuada de todos os profissionais do estabelecimento para compreensão do processo de doação de órgãos e tecidos.

A Organização de Procura de Órgãos (OPO) surgiu na instituição campo da pesquisa em 1998, baseada no Decreto $n^{\circ} 2268,2$ sendo definida como uma entidade sem fins lucrativos, com atuação regionalizada para detecção de doador potencial, constituído por um ou mais hospitais de sua área territorial de atuação.3 (Resolução $\left.n^{\circ} 103,1997\right)$.

A disparidade existente entre o número de doadores em potencial e o número real de doações já foi discutida por muitos autores. Segundo uma estimativa americana publicada em 1990, dos 20.000 indivíduos que morrem por ano e são doadores em potencial, apenas 3.000 realmente doam.

Noventa e cinco por cento dos doadores de órgãos em potencial encontram-se nas unidades de terapia intensiva.4 Baseados nestes dados, reconhecemos o importante papel dos profissionais de saúde que trabalham nessas unidades na relação de doadores em potencial e a situação final da doação.

Esperar o interesse de profissionais da área da saúde por uma 
mudança no processo de doação seria um tanto demorado, uma vez que a formação desses profissionais não exerce o mesmo apelo das inovações tecno-científicas de diagnóstico e tratamento.5 Por outro lado, esses são os indivíduos que poderão intermediar todo o processo de doação, e, no entanto, ainda falta-lhes informação.

Dentre os problemas enfrentados pela OPO no âmbito intrahospitalar, observou-se a carência de educação continuada dos profissionais em relação ao conhecimento sobre o processo de doação de tecidos e a existência de uma Organização de Procura de Órgão na Instituição, campo de estudo, ausência de fluxo para a notificação, baixo número de notificações de potencias doadores e a ocorrência esporádica de doação de córneas.

Nesse contexto, este estudo objetivou o desenvolvimento de uma sistemática para a doação intra-hospitalar de córneas, tendo como estratégia a capacitação de recursos humanos para favorecer o aumento de notificações de potenciais doadores de córneas para benefício da sociedade como bem social.

\section{OBJETIVOS}

Realizar o diagnóstico situacional do trabalho que vem sendo desenvolvido referente ao número de óbitos, notificações e doadores efetivos de córneas na Instituição;

Capacitar os colaboradores de áreas críticas para atuarem de forma mais efetiva no processo de doação intra-hospitalar de córneas;

Estabelecer fluxograma para a notificação intra-hospitalar de potencial doador de córneas e indicadores para levantamento, mensuração e análise dos resultados do projeto.

\section{MÉTODO}

Trata-se de um estudo descritivo, exploratório e documental, realizado numa instituição hospitalar pública de grande porte de referência em ensino e pesquisa na área cardiovascular da cidade de São Paulo, com aproximadamente 247 leitos, em fase de expansão. Formalmente, foi solicitado ao Comitê de Ética em Pesquisa o consentimento para a realização da coleta de dados, com aprovação sob n 3443 .

A população foi composta pela totalidade de questionários preenchidos pela equipe de enfermagem (enfermeiros e auxiliares de enfermagem) das áreas críticas. Do total de 192 (100\%) colaboradores, foi possível contar com 76 (39,58 \%).

Para o desenvolvimento deste trabalho foi realizado treinamento da equipe de enfermagem das áreas críticas no período de 08 a 12 de maio de 2006, em horários pré-estabelecidos e subdivididos para cada setor envolvido.

No treinamento, foi aplicado um questionário antes da aula, e, ao término, repetiu-se a aplicação do mesmo instrumento. Optamos por esse método visando a possibilidade de comparar o conhecimento em dois momentos e a compreensão do conteúdo exposto no treinamento. O programa de treinamento contém um questionário de avaliação do conhecimento do colaborador com questões fechadas relativas à categorização da equipe de enfermagem, ao processo de doação, captação de órgãos e notificação de córneas e o indicador de satisfação.
Para a apresentação dos resultados da avaliação do treinamento, as perguntas foram categorizadas como temáticas para posterior análise e planejamento das ações educativas, conforme Figura 1.

Figura 1: Categorização das perguntas por temáticas. São Paulo, 2007.

\begin{tabular}{|l|l|}
\hline Temáticas & Perguntas do questionário \\
\hline OPO & $\begin{array}{l}\text { Você sabe o que é OPO? Você sabe que no } \\
\text { hospital onde trabalha existe uma OPO? }\end{array}$ \\
\hline DOAÇÃO & $\begin{array}{l}\text { Você sabe como ser um doador de órgãos } \\
\text { e tecidos? Você sabe quais são os tipos de } \\
\text { doadores? Você sabe TODos os órgãos e } \\
\text { tecidos que podem ser doados? }\end{array}$ \\
\hline DIAGNÓSTICO & $\begin{array}{l}\text { Você sabe o que é morte encefálica? Morte } \\
\text { encefálica é igual ao coma? }\end{array}$ \\
\hline NOTIFICAÇÃO & $\begin{array}{l}\text { Você sabe quem notificar quando se tem } \\
\text { um potencial doador de múltiplos órgãos e } \\
\text { tecidos em seu setor? Você sabe a quem } \\
\text { notificar um potencial doador de córneas? }\end{array}$ \\
\hline MANUTENÇÃO & $\begin{array}{l}\text { Você sabe os cuidados que se deve ter para } \\
\text { a preservação das córneas de um portencial } \\
\text { doador? }\end{array}$ \\
\hline
\end{tabular}

\section{RESULTADOS}

Para o desenvolvimento da sistemática de doação intra-hospitalar de córneas foi realizado diagnóstico situacional, com coleta de dados no período de janeiro a dezembro de 2005, visando a comparabilidade no ano vigente ao da realização do projeto.

Apurou-se que em 2005 ocorreram 438 (100\%) óbitos na instituição campo de estudo, conforme registro do Serviço de Arquivo Médico (SAME); destes, 87 (20\%) foram notificados à OPO, efetivando-se a doação em apenas 14 (16\%).

Na seqüência, foram selecionadas as áreas (PS, UTI Pósoperatório, Unidade Coronária e UTI Crônico/Vascular) e a equipe (enfermagem) que compuseram a população do estudo, mediante o perfil da unidade, gravidade clínica do cliente internado e o estreito contato estabelecido entre os colaboradores e a OPO, sendo um facilitador da comunicação para notificar o óbito.

Do total de 192 (100\%) colaboradores de enfermagem, foi possível contar com a amostra de $76(39,58 \%)$.

Outra demanda da pesquisa foi a definição de um fluxograma de notificação de potencial doador de córneas (Figura 2) que

Figura 2: Fluxograma de notificação de potencial doador de córneas. São Paulo, 2007.

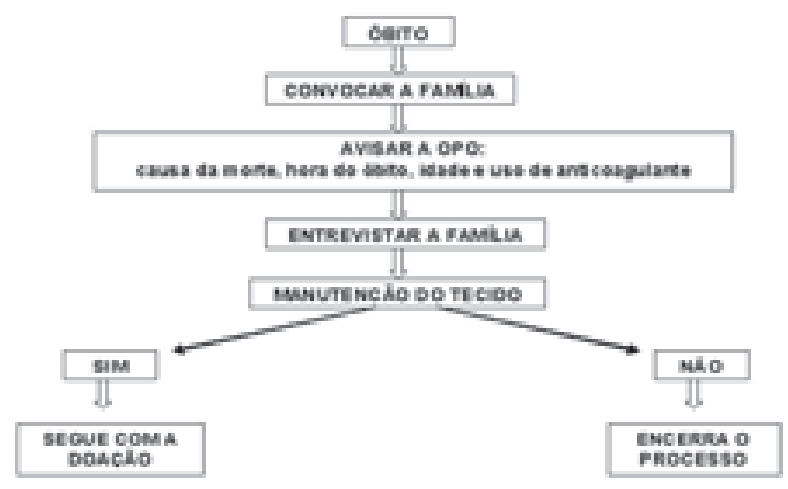


instrumentalizasse a equipe de enfermagem em tal atividade.

Foi ainda elaborado um instrumento de Busca Ativa IntraHospitalar de Potencial Doador de Córneas em todas as unidades de internação, uma vez que a ocorrência de óbito pode ser gerada em qualquer setor da Instituição, mesmo que a freqüência seja reduzida, quando comparada à ocorrência de óbitos nas áreas críticas.

Como recursos alternativos de comunicação visual foram desenvolvidos cartazes, adesivos para telefone, folders, ambiente virtual dentro do site institucional, para divulgação da OPO e sensibilização da comunidade interna.

Posteriormente, deu-se início ao treinamento dos colaboradores quanto ao processo de doação de órgãos e tecidos para transplantes, com o foco de atenção voltado para a notificação de potenciais doadores intra-hospitalares de córneas.

Como resultado encontrado exposto nas figuras 3 e 4, obtivemos um total de 760 (100\%) respostas relativas ao processo de doação, captação de órgãos e notificação de córneas, sendo que antes da aula ocorreu percentual de erro de 35,7 \% (271), enquanto após a aula, $3,9 \%$ (30), demonstrando que as dúvidas foram minimizadas por meio do conteúdo exposto, podendo ser observada a importância da orientação da equipe de enfermagem sobre o tema.

Figura 3: Distribuição do número de respostas erradas antes da aula, de acordo com as temáticas. São Paulo, 2007.

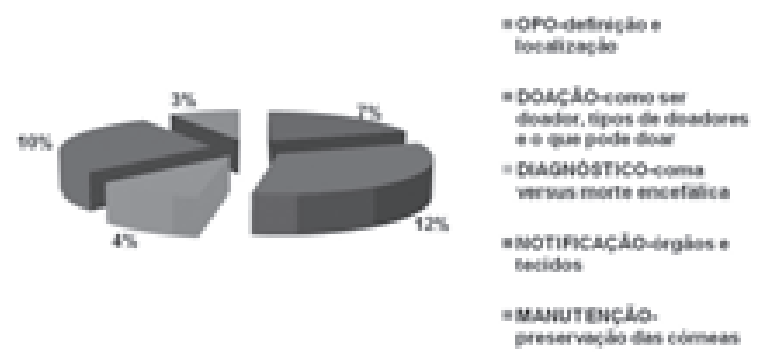

Figura 4: Distribuição do número de respostas erradas depois da aula, de acordo com as temáticas. São Paulo, 2007.

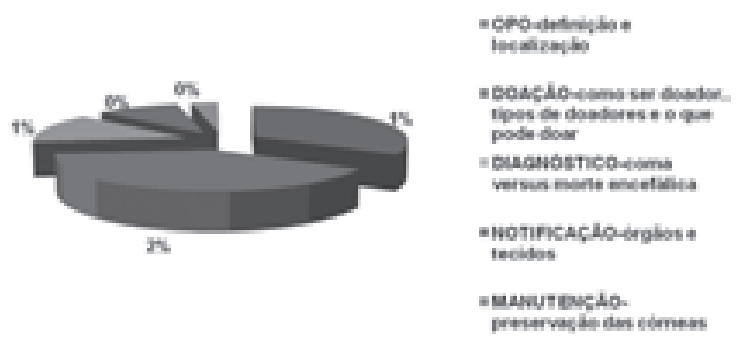

\section{DISCUSSÃO}

O conhecimento sobre o processo de doação foi objeto do estudo desenvolvido por Rodrigues,4 que avaliou o conhecimento e as atitudes dos médicos intensivistas sobre o processo de doação de córneas. Os depoimentos revelaram que dos 100 (100\%) médicos, 44 (44\%) não se achavam aptos a esclarecer as dúvidas de pacientes sobre a doação de córneas e que todos sentiam necessidade de ter atualizações nessa área, sendo que 96 (96\%) gostariam de receber essas informações por meio de folhetos e quatro (4\%) por palestras.
Com relação aos profissionais de enfermagem, verificamos na literatura que há carência inclusive no conhecimento da lei que rege a atividade de transplantes no Brasil por parte dos enfermeiros, como mostra o estudo de Roza.5

A análise do conhecimento de 50 profissionais de saúde de uma instituição de saúde do Rio de Janeiro sobre doação de órgãos confirmou o pressuposto que deu origem a este estudo, ou seja, foi constatado o desconhecimento dos profissionais da equipe de saúde sobre o processo de doação de órgãos para transplantes. 6

Com o estabelecimento da sistemática de notificação, o envolvimento e a contribuição efetiva da equipe de enfermagem a partir da capacitação em serviço, foi possível instituir o indicador de notificação e de doador efetivo de córneas (Tabela 1), a partir de maio de 2006, sendo mensalmente realizada a análise dos dados mensurados e dos resultados obtidos nesse intervalo de tempo (maio a dezembro de 2006), cujos dados foram compartilhados por todas as chefias médicas e de enfermagem envolvidas no processo.

Tabela 1: Indicador de notificação e de doador efetivo de córneas. São Paulo, 2007.

\begin{tabular}{|c|c|c|c|c|c|c|c|c|c|c|}
\hline \multirow[t]{2}{*}{ Setor } & \multirow[t]{2}{*}{ Meta } & \multicolumn{9}{|c|}{ Número de notificações/mês } \\
\hline & & Mai & Jun & Jul & Ago & Set & Out & Nov & Dez & Total \\
\hline Pronto Socorro & \multirow{11}{*}{ 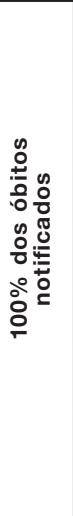 } & 13 & 18 & 19 & 15 & 15 & 5 & 7 & 5 & 97 \\
\hline Enfermaria I & & 1 & 1 & 2 & 0 & 0 & 2 & 3 & 5 & 14 \\
\hline Enfermaria II & & 0 & 1 & 0 & 2 & 0 & 0 & 0 & 0 & 3 \\
\hline $\begin{array}{l}\text { UTI - Crônico } \\
\text { e Vascular }\end{array}$ & & 3 & 9 & 12 & 11 & 13 & 5 & 5 & 3 & 61 \\
\hline $\begin{array}{l}\text { Centro } \\
\text { Cirúrgico I }\end{array}$ & & 0 & 0 & 0 & 1 & 0 & 1 & 2 & 1 & 5 \\
\hline $\begin{array}{l}\text { Centro } \\
\text { Cirúrgico II }\end{array}$ & & 0 & 0 & 1 & 1 & 1 & 1 & 0 & 0 & 4 \\
\hline $\begin{array}{l}\text { Unidade } \\
\text { Coronária }\end{array}$ & & 1 & 4 & 3 & 4 & 1 & 1 & 3 & 4 & 21 \\
\hline $\begin{array}{l}\text { UTI - Pós } \\
\text { operatório }\end{array}$ & & 6 & 4 & 6 & 7 & 3 & 7 & 3 & 2 & 38 \\
\hline Hemodinâmica & & 0 & 0 & 2 & 1 & 0 & 1 & 0 & 1 & 5 \\
\hline Pediatria & & 0 & 0 & 1 & 0 & 0 & 0 & 0 & 0 & 1 \\
\hline Ambulatório & & 0 & 0 & 0 & 0 & 0 & 0 & 0 & 0 & 0 \\
\hline \multicolumn{2}{|c|}{ Total de notificações } & 30 & 37 & 46 & 43 & 42 & 26 & 23 & 28 & 275 \\
\hline \multicolumn{2}{|c|}{ Número de óbitos } & 46 & 37 & 47 & 43 & 47 & 34 & 29 & 36 & 319 \\
\hline \multicolumn{2}{|c|}{ Não notificados } & 16 & 0 & 1 & 0 & 5 & 8 & 6 & 8 & 44 \\
\hline \multicolumn{2}{|c|}{ Doador efetivo } & 2 & 4 & 4 & 0 & 6 & 1 & 2 & 2 & 21 \\
\hline
\end{tabular}

Pode-se constatar o reflexo dessa sistemática no aumento do número de notificações em 2006, tendo alcançado 275 (86,2\%) notificações de potenciais doadores de córneas, na queda de 49,4 \% (44) no número de não-notificações e no aumento de $50 \%$ na doação de córneas, disponibilizando-se à sociedade 21 córneas, de acordo com o quadro abaixo.

A ação humanitária desenvolvida com este estudo implicou na redução da fila de receptores, uma vez que foram gerados como resultado 21 doadores efetivos de córneas, disponibilizando 42 córneas para o Estado de São Paulo.

\section{CONCLUSÃO}

O projeto nos permitiu transformar a realidade local a partir da sensibilização dos profissionais e da concretização do processo de doação intra-hospitalar de córneas. A pró-atividade dos profissionais e o empenho dos gestores das áreas com a questão da doação de córneas, 
por mais que seja incipiente, vem demonstrando a eficiência e o alcance dos objetivos propostos, principalmente no que diz respeito ao aumento do número de notificações e, por conseqüência, de doação de córneas. Os resultados positivos deste estudo podem ser associados à reflexão da sociedade sobre essa questão de saúde pública: a doação de órgãos e tecidos. A mídia, a legislação brasileira e os diversos grupos sociais estão atualmente discutindo sobre a problemática das filas de espera e as dificuldades de se encontrar um doador. Pode-se considerar que a incorporação de um novo fluxograma de notificação na rotina de trabalho e a falta de conhecimento dos profissionais de enfermagem foram os principais fatores dificultadores desse processo.

No entanto, apesar das dificuldades sentidas, percebeu-se o sucesso desta iniciativa. O programa demanda ainda monitoramento e investimento contínuo em ações que promovam a manutenção e melhoria dos resultados, uma vez que o maior desafio está pautado nos altos índices de não-notificação de potenciais doadores de córneas, que tendem a reaparecerem com o passar do tempo.

O desenvolvimento de parcerias com o gestor vigente, com os diretores das áreas administrativas do Instituto, sendo elas: Diretoria Administrativa, Financeira, Compras, Serviços Auxiliares, Administração da Fundação Adib Jatene, totalizando 20 participantes que não foram computados neste estudo e com a Central Nacional de Captação, Doação e Órgãos, foi uma estratégia utilizada pensando na operacionalização de todo processo, que trouxe contribuições para o trabalho diário dos enfermeiros da OPO-IDPC, fortalecimento das ações para o alcance dos objetivos e manutenção das propostas, como a elaboração de indicadores de qualidade, criação do fluxograma, análise crítica dos resultados alcançados e o desenvolvimento de planos de ação como forma de garantir a manutenção do processo e a busca de melhorias.

\section{ABSTRACT}

Purpose: This study aimed to develop a systematic for the in-hospital cornea donation, to promote an increase in the notifications of potential donors to benefit the society as a social asset. Methods: This is an experience report whose methodology was to make a punctual diagnosis on the amount of deaths, notifications, and effective cornea donors, training employees of critical areas in order to conduct the cornea donation process, establishing a notification schedule and to develop survey and measurement markers as well as analyzing the results. Result: The punctual diagnosis showed a low effective notification and cornea donation rate: among 438 (100\%) deaths, 87 (20\%) were reported to the OPO with only $14(16 \%)$ effective donations. The implementation of a notification schedule for potential cornea donors was adopted, as well as the training of 76 (39.58\%) professionals working in critical areas, with a $3.9 \%(30)$ after-class margin of error, showing that issues were minimized through the content exposed. In order to perform a monthly measurement, the amount of deaths, notifications and cornea donation was prepared as an instrument to the active search, which was shared by medical supervisors and the nursing staff involved in such process. Conclusion: The settlement of an internal flow, the involvement and effective contribution from the in-service nursing team and the training were reflected in an increase in the amount of notifications which attained 275 (86.2\%), meaning a 49.4\% (44) fall in the amount of non-notifications and a 50\% increase in the cornea donation which made available 21 donated corneas.

Keywords: Transplantation, In-service training, Nursing

\section{REFERÊNCIAS}

1. Portaria MS No 1.262 , de 16 de junho de 2006. Aprova o Regulamento Técnico para estabelecer as atribuições, deveres e indicadores de eficiência e do potencial de doação de órgãos e tecidos relativos às Comissões Intra-hospitalares de Doação de Órgãos e Tecidos para Transplante (CIHDOTT). Diário Oficial do Estado de São Paulo de 2006 - seção 1 - p.41

2. Decreto $N^{\circ}$ 2268, de 30 de junho de 1997. Regulamenta a Lei 9.434, de 4 de Fevereiro de 1997, que Dispõe Sobre a Remoção de Orgãos, Tecidos e Partes do Corpo Humano para Fins de Transplante e Tratamento e da Outras Providencias - seção 1 - p.24

3. Resolução SS-103 de 01 de agosto de 1997. Dispõe sobre a estrutura organizacional e operacional do Sistema Estadual de Transplante de São Paulo. Diário Oficial do Estado de São Paulo de 1997 - seção 1 - p. 15 a 20.

4. Rodrigues AM, Sato E. Entendimento dos médicos intensivistas sobre o processo de doação de córneas. Arq. Bras. Oftalmol. 2003;66(1):29-32.

5. Roza BA, Pellizetti N. Posicionamento de enfermeiros e médicos quanto à lei dos transplantes de órgãos. Acta Paul. Enferm. 2000;13(2):26-31.

6. Almeida IS de. Doação de órgãos: o que você sabe sobre esse tema? $57^{\circ}$ Congresso Brasileiro de Enfermagem [periódico na Internet]. 2005 Nov [acesso em 2009 Dez 12]. Disponível em: http://bvsms.saude.gov.br/bvs/publicacoes/57cbe/resumos/1063.htm 